\title{
Temas de Revisión \\ Detección de hongos y aflatoxinas en alimentos de importancia en salud pública
}

\author{
Detection of fungi and aflatoxins in food of importance in public
}

\author{
Jesús Rojas-Jaimes
}

\begin{abstract}
Resumen
Los mercados de abastos, localizados en diferentes distritos de Lima, son lugares que expenden una diversidad de alimentos a una gran población. Nuestro estudio determinó presencia de hongos y alflatoxina de importancia en Salud Pública en 25 alimentos de 15 mercados de 13 distritos de Lima. Entre los hongos de mayor importancia encontrados estuvieron Aspergillus niger en $V$. vinífera, Fusarium en $A$. hypogaea y Mucor en $O$. sativa y $C$. anuum. Entre las levaduras se identificó a $C$. tropicalis en $S$. lycopersicum, $C$. krusei en $D$. carota. Adicionalmente se identificó aflatoxinas carcinogénicas en los alimentos con mayor contaminación como Arachis hypogaea "mani" (149,7 ppb) del mercado Limoncillo/Rimac y Capsicum Chinense "ají panka" (56,4 ppb) del mercado Central de Comas. Por tanto, estos hallazgos generan un riesgo para presentar micosis superficiales y profundas, así como el riesgo de cáncer hepático, daño renal y del sistema inmunológico por aflatoximas en personas que manipulan y consumen estos alimentos respectivamente.
\end{abstract}

Palabras clave: Alimento, hongo, aflatoxinas, patógeno, micosis, regulación, seguridad alimentaria.

\section{Introducción}

Las infecciones micóticas representan un padecimiento frecuente a nivel mundial. Los casos de micosis superficiales son más de un billón, de candidiasis oral son dos millones, de candidiasis esofágica son más de un millón de casos anuales, el número de casos de aspergilosis es más de siete millones a nivel mundial (solo entre aspergilosis broncopulmonar alérgica y aspergilosis pulmonar crónica) y las micosis invasivas tienen aproximadamente 2 millones de casos anuales, principalmente causadas por el género Candida, Aspergillus y Pneumocystis jirovecii ${ }^{(1)}$. Siendo la población inmunosuprimida la más susceptible a estas ${ }^{(2-5)}$.

Los hongos de los géneros Aspergillus sp., Fusarium sp., Penicilium sp., Alternaria sp., y Claviceps sp., que colonizan granos y frutos producen metabolitos carcinogénicos, inmunosupresores, neurotoxinas y son alérgicos para la población humana ${ }^{(6)}$.

Las micotoxinas son producidas por dos géneros de hongos filamentosos Aspergillus y Penicillium. Las especies productoras son: Aspergillus flavus, Aspergillus parasiticus, Penicillium puberalis y Aspergillus oryzae. En 1967, Nulik y Holiday reportaron también a las especies Aspergillus niger, Aspergillus gruber, Aspergillus wentii, Penicillium frecuentans, Penicillium variable y Penicillium citrinum $^{(7)}$. Estos hongos son contaminantes habituales de alimentos como granos y cereales almacenados, cuyas condiciones óptimas para la producción de micotoxinas son una temperatura de $25{ }^{\circ} \mathrm{C}$ y una humedad relativa del $95 \%$. Las principales toxinas producidas por este género de mohos son las aflatoxinas y las ocratoxinas ${ }^{(8,9)}$.

Las aflatoxinas: son micotoxinas cancerígenas, teratogénicas, mutagénicas, que tienen tropismo por órganos como hígado, cerebro y riñón. Estas toxinas son producidas bajo ciertas condiciones de temperatura y humedad por $A$. flavus y $A$. parasiticus y su síntesis está regulada por los genes (AFLR y AFLS). Se han descrito 18 tipos de esta micotoxina donde se destacan B1, B2, G1, G2, M1, M2. E1 metabolito tóxico más importante de este grupo es la aflatoxina $\mathrm{B} 1^{(10,11)}$

El objetivo del presente estudio fue identificar los hongos y aflatoxinas de importancia en salud pública en alimentos de consumo masivo en los principales mercados de abastos de Lima Metropolitana.

\section{Metodología \\ Área de estudio}

El universo de trabajo estuvo constituido por alimentos de consumo humano que se obtuvieron de 15 mercados de abastos de 13 distritos de Lima, distribuidos entre los diferentes sectores socioeconómicos, en los distritos: Breña, Cercado de Lima, El Agustino, Independencia, Jesús María, La Victoria, Lince, Rímac, San Borja, San Martín de Porras, Surquillo, San Juan de Miraflores y Comas.

${ }^{1}$ Universidad Científica del Sur, Escuela de Medicina Humana, Lima-Perú. 


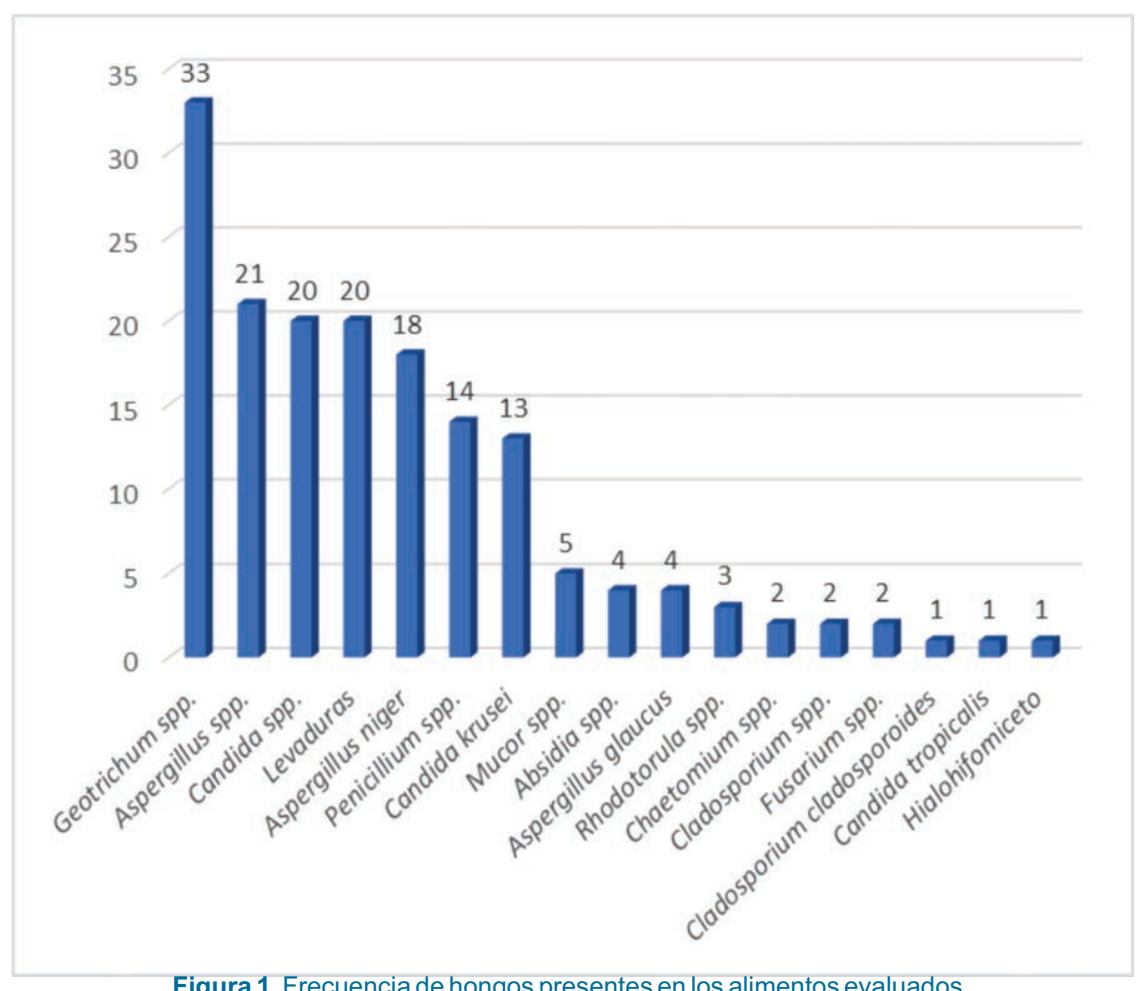

\section{Material de Estudio}

- Cereales: Arroz (Oryza sativa), maíz "Variedad Saccharata" (Zea mays), trigo (Triticum spp), cebada (Hordeum vulgare) y cebada tostada.

- Leguminosas: Frijol (Phaseolus vulgaris), maní (Arachis hypogaea) y maní tostado, arveja (Pisum sativum) y alberja seca, lentejas (Lens culinaris), frijol "Canario" (Phaseolus vulgaris L.), frijol "Castilla" (Vigna unguiculata).

- Solanáceas: Papa (Solanum tuberosum), tomate (Lycopersicon lycopersicum), páprika (Capsicum annuum), rocoto (Capsicum pubescens), Pimientón (Capsicum annuum L), ají colorado (Capsicum chinense), ají colorado variedad "Panca", ají "Amarillo" (Capsicum baccatum).

- Otros: Habas (Vicia faba) y habas frescas, pasas (Vitis vinifera), zanahoria (Daucus carota).

De forma aleatoria fue recolectada 195 muestras de $250 \mathrm{~g}$ de los mercados de abastos ya mencionados.

\section{Procesamiento y aislamiento de hongos patógenos}

Las muestras fueron recogidas en bolsas plásticas de cierre hermético de los diferentes puntos de toma de muestras. Se procesó $60 \mathrm{~g}$ de alimentos en licuadora-trituradora con vaso para granos con cuchilla especial y de esta molienda se sacó 10 g y se colocó con paletas de acero en frascos estériles. Después de cada procedimiento se lavó con agua todos los materiales en contactos con alimentos, luego se desinfecto con etanol de $96^{\circ}$ y se procedió al secado con papel toalla

Se tomaron $500 \mu \mathrm{l}(1 / 2 \mathrm{ml})$ del licuado y se sembraron por agotamiento en dos tubos con agar glucosado Sabouraud sin antibióticos y anti fúngicos; paralelo se hizo montaje húmedo en lámina y coloración de azul de metileno.

La identificación de los hongos patógenos filamentosos y de las levaduras se realizó por metodología convencional manual usando los métodos micológicos.

\section{Preparación e identificación de Aflatoxinas}

Se pesó $5 \mathrm{~g}$ de la muestra y se mezcló con $25 \mathrm{ml} \mathrm{de}$ metanol / agua destilada (70/30, v/v), agitándose por 10 minutos a temperatura ambiente $\left(20-25^{\circ} \mathrm{C}\right)$. Luego, el extracto se filtró a través de un papel Whatman $\mathrm{N}^{\circ} 1$.

Luego se diluye $250 \mathrm{uL}$ del filtrado con $1.5 \mathrm{ml}$ de agua destilada. Se utilizó 50 ul del filtrado diluido por micropozo en el test. Finalmente para identificar las aflatoxinas se utilizó el método de Veratox ${ }^{\circledR}$ Aflatoxina total.

\section{Resultados}

\section{Prevalencia de hongos identificados}

Los hongos más frecuentemente encontrados en distintos alimentos y mercados fueron Geotrichum spp 


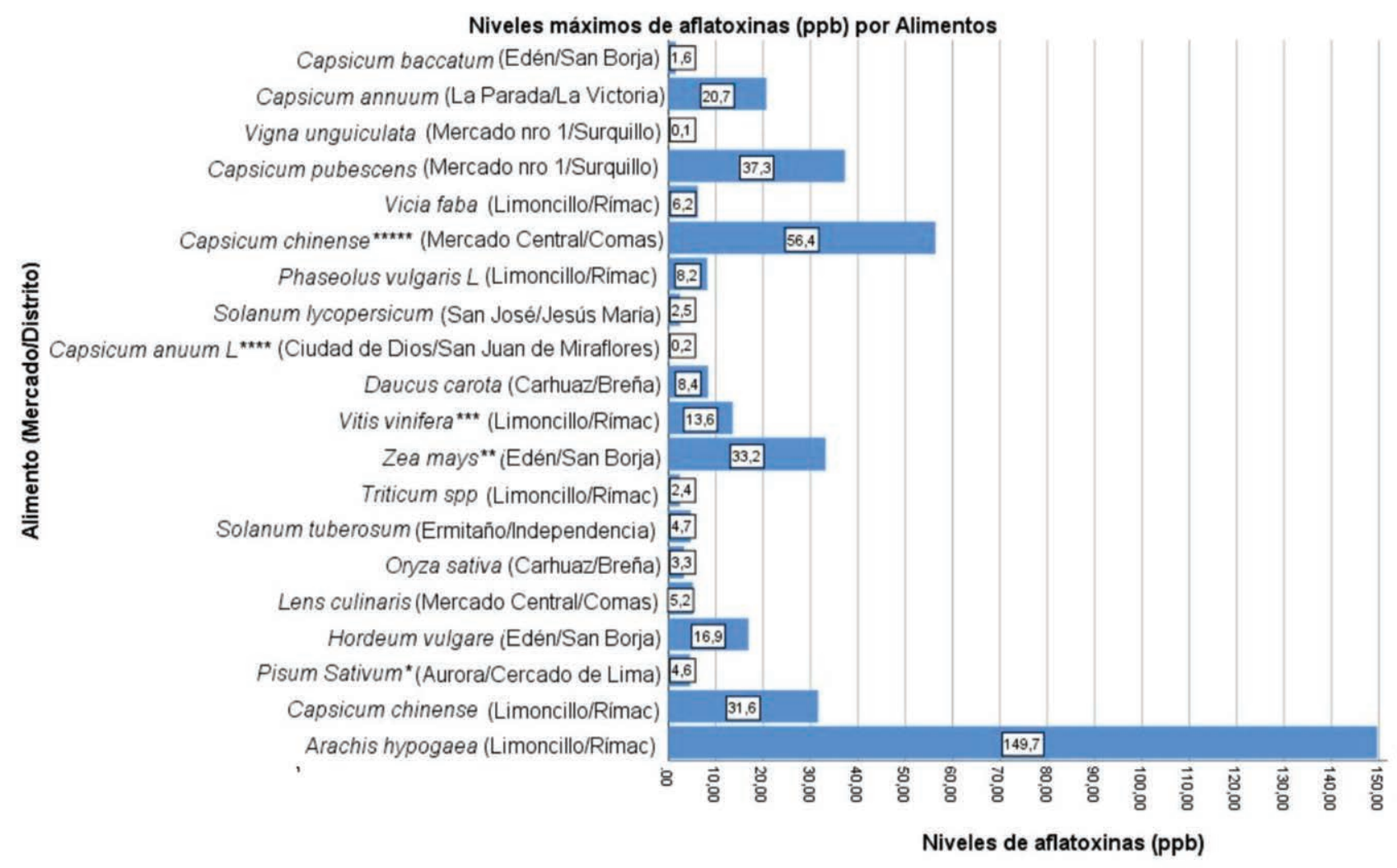

\begin{tabular}{|l|l|}
\hline Leyenda \\
\hline Capsicum chinense \\
\hline Capsicum anuum & Variedad de Aji Colorado \\
\hline Vitis vinifera & Pimientón \\
\hline Zea mays mat $^{* \star \star}$ & Pasas \\
\hline Pisum sativum & Mote \\
\hline
\end{tabular}

Figura 2. Niveles máximos de aflatoxinas en los alimentos que se expenden en mercados de Lima Metropolitana.

$(\mathrm{n}=33)$, Aspergillus spp $(\mathrm{n}=21)$, Candida spp $(\mathrm{n}=20)$, Levaduras no identificables $(\mathrm{n}=20)$, Aspergillus niger $(\mathrm{n}=18)$, Penicillium spp $(\mathrm{n}=14)$ y Candida krusei $(\mathrm{n}=13)$. Los demás aparecieron en menos de 6 cultivos, incluyendo hongos oportunistas como Mucor spp, Rhodothorula spp, Fusarium spp, Cladosporium spp, Cladosporium cladosporoides y Candida tropicalis (Figura 1).

\section{Niveles máximos de aflatoxinas por alimentos}

Los alimentos que pasaron los 20ppb equivalente a $\mathrm{ug} / \mathrm{Kg}$ de aflatoxinas fueron: Arachis hypogaea-Mercado Limoncillo/Rimac, Capsicum chinense**. Mercado Central/Comas, Capsicum pubescens- Mercado \# 1/Surquillo, Zea mays- Mercado Eden/ San Borja, Capsicum chinense- La Chira/Rímac, y Capsicum annuum- La Parada/La Victoria en orden decreciente respectivamente. Los demás alimentos tuvieron limites máximos de aflatoxinas por debajo de 20ppb (Figura 2).

\section{Discusión}

En el estudio de hongos en alimentos en 13 mercados de abastos de Lima Metropolitana, en diferentes sectores socioeconómicos y en los tres puntos geográficos (norte, centro y sur) se identificó hongos patógenos, entre los cuales destacan como hongos patógenos capaces de causar enfermedad al ser humano, filamentosos Aspergillus niger en $V$. vinifera, Fusarium en $A$. hypogaea y Mucor en $O$. sativa y $C$. anuum. Entre las levaduras se identificó a $C$. tropicalis en $S$. lycopersicum, C. krusei en D. carota. Es importante mencionar que algunos de los alimentos, con prevalencia de hongos patógenos, se consumen crudos como $V$. vinífera, $C$. anuum, $S$. lycopersicum y $D$. carota, contrastando estos hallazgos con la patogenia que presentan los hongos en estudio $^{(1)}$. Adicionalmente se observó que 6 tipos de alimentos muestreados en los mercados de abastos de 13 distritos de Lima superaron los límites permisibles de la micotoxina carcinogénica "aflatoxina" lo cual sería un riesgo 
para la Salud Pública. Entre los alimentos con mayores valores de contaminación estuvieron Arachis hypogaea "maní" del mercado Limoncillo/ Rimac y Capsicum Chinense "ají panka" del mercado Central de Comas. Aunque en estudio previos se ha presenciado niveles altos de aflatoxinas en granos, nuestro estudio destaca el encontrar también contaminación en frutos que se consumen no cocidos y genera un alto riesgo en la salud publica ${ }^{(8-11)}$.

\section{Conclusión}

Se concluye que se observó la presencia de hongos filamentosos, levaduras y aflatoxinas en los alimentos evaluados que se comercializan para el consumo. Por tanto, se de importancia capacitar a las personas responsables en la cadena de producción y comercialización de alimentos para la prevención, disminución y neutralización de las aflatoxinas y la prevención por contaminación de hongos patógenos debiendo involucrar a organismos como el Servicio Nacional de Sanidad Agraria que debe identificar los contaminantes en los alimentos de consumo público, Ministerio de Salud que debería inspeccionar los alimentos a través de estudios microbiológicos la correcta inocuidad de los alimentos y Ministerio de Agricultura que debería monitorear y capacitar para la correcta siembra y producción de los alimentos para evitar la contaminación por hongos y sus metabolitos .

\section{Referencias bibliográficas}

1. Bongomin F, Gago S, Oladele RO, Denning DW. Global and Multi-National Prevalence of Fungal Diseases-Estimate Precision. Journal of fungi. 2017;3(4).

2. Talle M, Hamidu I, Nasir I-A, Mursal A, Dikwa K, Jelili M, et al. Prevalence and profile of pulmonary fungal pathogens among HIV-infected patients attending University of Maiduguri Teaching Hospital, Nigeria. The Egyptian Journal of Internal Medicine. 2017;29(1):11-15.

3. Rubaihayo J, Tumwesigye NM, Konde-Lule J, Wamani H, Nakku-Joloba E, Makumbi F. Frequency and distribution patterns of opportunistic infections associated with HIV/AIDS in Uganda. BMC research notes. 2016;9(1):501.

4. de Oliveira RB, Atobe JH, Souza SA, de Castro Lima Santos DW. Epidemiology of invasive fungal infections in patients with acquired immunodeficiency syndrome at a reference hospital for infectious diseases in Brazil. Mycopathologia. 2014;178(1-2):71-8.

5. Kaur R, Dhakad MS, Goyal R, Bhalla P, Dewan R. Spectrum of Opportunistic Fungal Infections in HIV/AIDS Patients in
Tertiary Care Hospital in India. The Canadian journal of infectious diseases \& medical microbiology. Journal canadien des maladies infectieuses et de la microbiologie medicale. 2016;2016:2373424.

6. Antón A, Lizaso J. Hongos y Micotoxinas. Fundación Ibérica para la Seguridad Alimentaria. 2001:30(1):1-25

7. Bolet M, Socarrás MM. Micotoxinas y cáncer. Revista Cubana de Investigación Biomédica. Cuba 2004.

8. Gimeno A, Martins ML. Micotoxinas y micotoxicosis en animales y humanos. 3 edición. 2011.

9.Segal BH. Aspergillosis. N Engl J Med.2009;360(18):187084.

Shan X, Williams WP. Toward elucidation of genetic and functional genetic mechanisms in corn host resistance to Aspergillus flavus infection and aflatoxin contamination. Front Microbiol. 2014;5:364.

11. Medina A, Rodriguez A, Magan N. Effect of climate change on Aspergillus flavus and aflatoxin B1 production. Front Microbiol. 2014;5:348.

Citar como: Rojas-Jaimes J. Detección de hongos y aflatoxinas de importancia en salud publica en alimentos. Diagnóstico(Lima).2019;58(2):97-100. DOI: https://doi.org/10.33734/diagnostico.v58i2.213

Correspondencia: Jesús Rojas-Jaimes Dirección: Carretera Panamericana Sur 19, Villa El Salvador 15067 Teléfono: 993638840

Correo electrónico: jesus.rojas.jaimes@gmail.com 